\title{
On Irreversible Investment
}

\author{
FRANK RIEDEL \\ Dept. of Economics \\ University of Bonn \\ Adenauerallee 24-26 \\ D-53113 Bonn \\ Germany \\ frank.riedel@uni-bonn.de
}

\author{
$\mathrm{Xia} \mathrm{Su}$ \\ Bonn Graduate School of Economics \\ University of Bonn \\ Adenauerallee 24-26 \\ D-53113 Bonn \\ Germany \\ xia.su@uni-bonn.de
}




\begin{abstract}
This paper develops a general theory of sequential irreversible investments in capital where a firm has the option to expand its current capacity or just wait for better time. Facing economic uncertainty, the firm has an operating function of the current capacity and an exogenous stochastic factor modelled by semimartingale. This general model encompasses all previously studied models, including the deterministic case as well as the stochastic case with Geometric Brownian motions, Lévy processes and even with regime shift. In this paper, general existence and uniqueness results are first provided for irreversible investments with finite and infinite horizon, respectively. As the main contribution of this paper, a new method is proposed to characterize the optimal investment policy, the base capacity policy. Under the policy, the capacity is kept always at or above the base capacity which is characterized by a stochastic backward equation. This new method gives a number of new qualitative insights into the nature of the irreversible investment. It is demonstrated that the optimal policy equals the marginal operating profit and the user cost of capital in those free intervals when the irreversibility constraint does not bind. While, the equality holds true only on average in block intervals when no investment occurs. Besides, this method easily leads to some general comparative statics results: When the operating profit function is supermodular, the base capacity increases monotonically with the exogenous shock; and the firm size always declines with the user cost of capital. Finally, explicit solutions are derived when the exogenous economic shocks are modelled by Lévy processes and the operating profit function is of Cobb-Douglas style.
\end{abstract}

Key words and phrases: Sequential Irreversible Investment, Capacity Choice, Lévy Processes.

JEL subject classification: C61, D81, E22, G11 


\section{Introduction}

Built on the pioneering works by Jorgenson (1963) and Arrow (1968), an extensive literature investigates the irreversible investment problem under uncertainty. As one of the significant issues, the timing of irreversible investment problems is determined via different approaches. Traditionally, the Net Present Value (NPV) method gives the investment strategy that investment is made if and only if the NPV is non-negative after defining the net present value as the difference of the expected present value of future cash flows and that of costs. As widely acknowledged in the literature, this conventional rule has to be revised since it considers the investment only as being "now or never". In fact, the investment decision is greatly affected by three factors: uncertainty over the future economic environment, cost irreversibility in the sense that the investment expenditure can be only partially or not at all recovered, and some degree of flexibility required in the timing of investment.

With the appearance of the real options theory, their implication on irreversible investments are well emphasized. In the standard real option model, the firm who has an investment opportunity is holding an option on real assets. Then, an investment decision problem is equivalent to exercising an American option. Consequently, investment is undertaken if and only if the net present value exceeds the option premium of waiting. In the simple model of a single project, the optimal investment strategy is derived by means of the standard real options approaches, i.e., contingent claim analysis and dynamic programming method, as fully exploited in Dixit and Pindyck (1994). These techniques give elegant solutions: The optimal investment policy is a trigger strategy such that the investment is initiated at the first time when the value of the investment project comes to a critical threshold.

However, capacity is usually built up gradually over time rather than once at time. As uncertainty prevents the firm from one-shot investment when taking into consideration the possibility of economic troughs. On the other hand, uncertainty also creates new investment opportunities (cf. Henry (1974), Arrow and Fisher (1974)). In this case, the single investment model is relatively restricted and not that relevant to the reality. Thus, one

concern becomes increasingly important in the literature on the incremental capital expansion, or sequential irreversible investments in capacity. 
Arrow (1968) first comes up with sequential irreversible investment problems under certainty. The problem is formulated in a continuous-time but deterministic optimization model with a deterministic interest rate and a profit function which does not incorporate uncertain economic shocks. The same problem but under uncertainty is studied in Pindyck (1988) by the contingent claim analysis. Adapting the technique for the single investment to sequential irreversible investment problems, Pindyck (1988) considers the marginal investment decision. Rather than focusing on how much to invest at each time, he identifies the timing of the infinitesimal stock of capital. Generally, models of irreversible investments under uncertainty assume that the firm is subject to a multiplicative economic shock that evolves according to a Geometric Brownian Motion with constant drift and volatility (e.g., Bertola (1989), Bertola (1998), Pindyck (1988) and Kobila (1993)). In their models, either a Cobb-Douglas or a general (see Kobila (1993)) operating profit function is assumed. Boyarchenko (2004) extends the capital expansion model to the case where economic shocks are characterized by exponential Lévy processes. An interesting extension by Guo, Miao, and Morellec (2005) concerns regime shifts where the drift and volatility of the Brownian motion switch between different states according to a continuous time Markov chain.

This paper also develops a framework to study sequential irreversible investments in capital when the firm faces uncertain economic situations. For this purpose, a very general model is constructed where a value-maximizing firm is facing with a concave operating profit function and subject to multiplicative economic shocks modelled by any stochastic processes. It covers not only all the models studied before but also the standard finance model where the uncertainty is characterized by a semi-martingale process. Given the general model, the existence and uniqueness theorem is first developed, which is not yet available in the literature. Sufficient conditions are provided such that a unique optimal investment policy always exists for finite and infinite horizon cases, respectively.

Then, the optimal investment rule is derived in a very detailed and intuitive way by a new method. By means of this method, the sequential irreversible investment problem is treated as a sequence of singular control problems. As the starting point, the necessary and sufficient optimality conditions are first derived by investigating the relationship of the marginal 
profit and cost of investment. In principal, the marginal profit by installing any infinitesimal unit of capital has to be lower than or equal to the cost. Investment occurs "at the margin" if and only if the capacity is depreciated or the investment cost declines such that the marginal profit becomes equal to the cost. Then, the marginal investment problem is solved by constructing and tracking a base capacity and calculating the resulted stochastic backward equation. After achieving the base capacity, the optimal investment policy is determined: if the current capacity is lower or equal to the base capacity, investment is undertaken at once to increase the capacity to the base capacity; otherwise just keep the current capacity.

One advantage of this method is that it allows a qualitative general characterization of the optimal investment. A thorough analysis is especially carried out in this paper with illustrative examples. Meanwhile, the obtained implications are compared with those of Arrow (1968) for sequential irreversible investments under certainty. Generally, the optimal investment plan can be characterized by three different phenomena: smooth continuous investment, lump sum investment and singular investment. It is demonstrated that the marginal profit is indeed equal to the user cost of capital in the free interval where the smooth investment occurs at a positive rate, as in the case of reversible investments. Lump sum investment is possible only with information surprises in the blocked interval. In this interval, the equality of marginal profit and the user cost of capital is however maintained only in expectation on average over time. The remaining investment phenomenon is defined as singular investment that takes place continuously but without rate of investment. It exits whenever the uncertainty is (partly) modelled by Brownian motions.

Moreover, this new method easily leads to some general comparative statics results. First, the base capacity is shown to be monotonically increasing in the exogenous shock when the operating profit function is supermodular or equivalently has increasing differences in capacity and exogenous shock. Besides as well-known in the literature, the firm size always declines with the user cost of capital.

Generally, numerical methods have to be used to identify the base capacity according to the algorithm given in the paper. Nevertheless, closed-form solutions of the optimal investment policy are possible to some 
specific cases, which is another contribution of this paper. To emphasize this feature, a specific model is constructed on the basis of Pindyck (1988) and Abel and Eberly (1996). In the model, an infinitely-lived firm is endowed with the operating profit function of Cobb-Douglas style. Specially, multiplicative economic shocks are modelled by Lévy processes with possible rare and unexpected jumps. Under this construction, the base capacity is characterized simply by a factor $\kappa$ times the economic shocks.

The remainder of this paper is organized as follows. Section 2 presents a rather general model to study the optimal timing problem of sequential irreversible investments in capital. General existence and uniqueness results are first developed in this paper and given with a detail proof in Appendix. Section 3 provides a clear and intuitive derivation of the optimal investment policy. In addition, a qualitative analysis is made on the property of the irreversible investment in Section 4. Section 5 gives some general comparative statics results for the irreversible investment problem. Particularly, an explicit solution is given in Section 6 for the case that the firm is endowed with a Cobb-Douglas operating profit function and subject to economic shocks modelled by an exponential Lévy process. Finally, Section 7 concludes the paper with a short summary and remark.

\section{$2 \quad$ Irreversible Investment Model}

To develop the sequential irreversible investment theory, a general model is first set up, which covers all existing models in the literature. In addition, this section investigates the existence and uniqueness of the irreversible investment decision problem. Sufficient conditions are provided for the existence and uniqueness of an optimal investment plan both for finite and infinite horizon cases.

\subsection{Irreversible Investment Model Setup}

Consider a firm that chooses a dynamic capacity expansion plan over a time horizon $\hat{T} \leq \infty$ which can be finite or infinite. It produces output with only one type of capital stock $C$. At each moment of time $t \in[0, \hat{T}]$, the operating profit is given by a function $\pi\left(X_{t}, C_{t}\right)$ of current capacity $C_{t}$ and some exogenous state variable $X_{t}$ with real values. Intuitively, $X_{t}$ can be 
regarded as multiplicative economic shocks, reflecting the changes in, e.g., technology, demand conditions, information leakage and so on which have direct or indirect effects on the firm's production. The stochastic process $\left(X_{t}\right)_{t \in[0, \hat{T}]}$ is formally defined on some underlying filtered probability space $\left(\Omega, \mathcal{F}_{T},\left(\mathcal{F}_{t}, 0 \leq t \leq \hat{T}\right), \mathbb{P}\right)$ satisfying the usual conditions of right-continuity and completeness and that $\mathcal{F}_{0}$ is $\mathbb{P}$-a.s. trivial. Suppose in addition that $\pi: \mathbb{R} \times \mathbb{R}_{+} \rightarrow \mathbb{R}_{+}$is strictly increasing and strictly concave in capacity $C$ with derivative $\pi_{c}(x, c)$ that satisfies the Inada conditions

$$
\lim _{c \rightarrow 0} \pi_{c}(x, c)=\infty
$$

and

$$
\lim _{c \rightarrow \infty} \pi_{c}(x, c)=0
$$

for all $x \in \mathbb{R}$. Moreover, there are no costs as long as no investment is made, or equivalently, $\pi(0)=0$.

As assumed in Arrow (1968), the price of capital goods used to build up capacity is taken as the numéraire. Thus, the cost of investment is always 1 and the short-term interest rate at time $t, r_{t}$, is expressed in terms of the capital goods but not money. Formally, $\left(r_{t}\right)_{t \in[0, \hat{T}]}$ is a nonnegative bounded optional process.

Given the operating profit, the firm chooses a plan $I=\left(I_{t}\right)_{t \in[0, \hat{T}]}$ of cumulative investments, a right-continuous adapted process with $I_{0-}=0$. As investment is irreversible (That is, installed capital has no resale value.), $I$ has to be nondecreasing. The investment plan leads to a capacity $C^{I}=\left(C_{t}^{I}\right)_{t \in[0, \hat{T}]}$ that starts in $C_{0_{-}}^{I}=0$ and evolves according to the differential equation

$$
d C_{t}^{I}=d I_{t}-\delta_{t} C_{t}^{I} d t
$$

for some depreciation rate $\left(\delta_{t}\right)_{t \in[0, \hat{T}]} \geq 0$, a nonnegative bounded optional process. An investment plan $I$ is admissible if its net present value is finite, i.e.,

$$
\mathbb{E}\left[\int_{0}^{\hat{T}} e^{-\int_{0}^{t} r_{s} d s} d I_{t}\right]<\infty .
$$

In this context, the firm is designed to maximize the expected present value 
of the future overall net profits

$$
\Pi(I)=\mathbb{E}\left[\int_{0}^{\hat{T}} e^{-\int_{0}^{t} r_{s} d s}\left(\pi\left(X_{t}, C_{t}^{I}\right) d t-d I_{t}\right)\right]
$$

over all admissible plans $I$. Note that the net profit $\Pi(I)$ is well defined for all admissible plans but potentially infinite. In the next subsection, some conditions are to be given to ensure finiteness.

Before solving the sequential irreversible investment decision problem with a new method, all examples studied so far that are included in our setup are first indicated as follows.

Example 2.1 The general setup includes the deterministic case with an arbitrary deterministic interest rate $r$ and the operating profit $\pi\left(t, C_{t}\right)$ (Thus, time is the state variable, namely, $\left.X_{t}=t\right)$. This case has been fully analyzed by Arrow (1968) in complete generality by using the calculus of variations, in particular Pontryagin's principle.

Example 2.2 The best studied special case under uncertainty has a separable operating profit function $\pi(x, c)=e^{x} \pi(c)$ and an infinite time horizon. and Pindyck (1988) characterize $X$ as a Brownian motion (BM),

$$
X_{t}=\mu t+\sigma W_{t}
$$

where $W_{t}$ is the standard $B M, \mu$ and $\sigma$ are the constant drift and volatility, respectively. Moreover, they assume a constant interest rate $r_{t}=r, \forall t \in$ $[0, \infty]$, and a Cobb-Douglas operating profit function $\pi(c)=\frac{1}{1-\alpha} c^{1-\alpha}$ for some $0<\alpha<1$. Boyarchenko (2004) allows $X$ to be a Lévy process, a Markov process with independent and identically distributed increments. An interesting extension concerns regime shifts where the parameters of the BM switch between different states according to a continuous time Markov chain, see Guo, Miao, and Morellec (2005). Kobila (1993) presents the general dynamic programming approach for nonseparable operating profit and diffusion state variables.

\subsection{Existence and Uniqueness}

Although a number of explicit case studies have been carried out, no general existence and uniqueness theorem is available in the literature. The present 
subsection provides conditions that ensure existence and uniqueness of a solution for the case of a finite horizon. Those for an infinite horizon are provided in Appendix $A$.

Take an auxiliary function as the starting point. Due to the assumptions of the operating profit function $\pi$, the indirect operating profit function

$$
\pi^{*}(x, r, \delta)=\max _{c \geq 0} \pi(x, c)-(r+\delta) c
$$

exists for fixed parameters $x, r, \delta \in \mathbb{R}$. The unique maximizer is given by $c^{*}(x, r, \delta)$ that solves the first order condition

$$
\pi_{c}(x, c)=r+\delta .
$$

Remark 2.3 This auxiliary function describes the optimal capacity under perfect reversibility of investment, the myopic decision rule. In this case, marginal profit has to be equal to the user cost of capital 1 which is given in this paper by the sum of interest and depreciation rate. Please compare with the discussion below.

Assumption 2.4 (i) $\mathbb{E}\left[\pi^{*}\left(X_{t}, r_{t}, \delta_{t}\right)\right]<\infty \forall t \in[0, \hat{T}]$,

(ii) $K \triangleq \mathbb{E}\left[\sup _{t \leq \hat{T}} c^{*}\left(X_{t}, r_{t}, \delta_{t}\right)\right]<\infty$.

The assumption ensures that the reversible investment problem has a finite value and that the overall maximum of optimal reversible capacity is integrable. On this basis, uniqueness and existence is achieved as stated in the following theorem ${ }^{2}$,

Theorem 2.5 Under Assumption 2.4, there always exists a unique optimal irreversible investment plan $I^{*}$.

\footnotetext{
${ }^{1}$ As first defined by Jorgenson (1963), the user cost of capital is in the standard neoclassical economics the opportunity cost of holding one unit of capital for a period. It consists of three components: the financial cost of the capital measured by the interest rate $r$, the depreciate $\operatorname{cost} \delta$ and the lost gain in the value of that unit of capital $\frac{E\left[d P_{t}\right]}{P_{t}}$ where $P_{t}$ denotes the purchasing price of the capital.

${ }^{2}$ The proof is given in Appendix A.1.
} 
Example 2.6 The benchmark example in the literature has the operating profit function $\pi(x, c)=e^{x} \frac{c^{1-\alpha}}{1-\alpha}$ with a constant parameter $\alpha>0$. If $X$ is a BM of the form $X(t)=\mu t+\sigma W(t)$, Assumption 2.4 is satisfied. More generally, Assumption 2.4 holds true for for Lévy processes without positive jumps.

Proof: The maximizer of (3) is obtained as

$$
c^{*}(x, r, \delta)=\frac{1}{(r+\delta)^{\frac{1}{\alpha}}} e^{\frac{x}{\alpha}}
$$

Then, it gives the optimal indirect operating profit

$$
\pi^{*}(x, r, \delta)=\frac{\alpha}{(1-\alpha)(r+\delta)^{\frac{1-\alpha}{\alpha}}} e^{\frac{x}{\alpha}}
$$

Thus, it is enough to show that the running maximum $Z=\sup _{t \leq \hat{T}} X_{t}$ satisfies $\mathbb{E}\left[e^{\lambda Z}\right]<\infty$ for all positive $\lambda>0$. As is well-known, it always holds true for BMs and more generally for Lévy processes without positive jumps (see Bertoin (1996), Chapter VII).

\section{Optimal Irreversible Investment Policies}

The objective of the firm is to find out the contingent investment rule $\left(I_{t}^{*}\right)_{t \in[0, \hat{T}]}$ and the corresponding capital stock $\left(C_{t}^{*}\right)_{t \in[0, \hat{T}]}$ such that Equation (2) obtains the maximum. For comparison purposes, we first introduce briefly the "myopic" investment rule when investment is completely reversible. Then, the optimal irreversible investment policy is derived in a detailed and intuitive way by a new method.

\subsection{Reversible Investment Policies}

Standing as the opposite case to irreversibility, capital investment is perfectly reversible if capital goods can be rented freely in the market. In this case, the firm can adjust capacity at no cost at every point of time. As shown by Jorgenson (1963), also Arrow (1968) and the literature therein, the investment criterion is to equate marginal operating profit and the user cost of capital, i.e.,

$$
\pi_{c}\left(X_{t}, C_{t}^{I}\right)=r+\delta
$$


The firm equates the marginal profit from capacity to the cost of renting a further marginal unit for an infinitesimal period. This cost is given by the interest rate augmented by the cost of replacing the depreciated amount of capacity. In general, the change of the price of capacity enters as well. As the price of the capital goods is taken as the numéraire here, the change is always zero.

The optimal investment plan has a special "myopic" property in the sense that future expected marginal profits do not appear in Eqn. (4). Of course, this does not mean that the firm is myopic, though. The optimal plan does not consider future marginal profits since the firm can resize its capacity in any desired way by purchasing or selling the capital.

\subsection{Irreversible Investment Policies}

Once investment is irreversible, the optimal investment plan is greatly affected since the firm has to account for the effect of capital stock on future marginal profits. This subsection studies the problem by a new method which works for a more general class than those studied before, including the standard finance models where uncertainty is modelled by a semi-martingale. Meanwhile, the method incorporates economic intuition into the derivation and allows a general qualitative characterization of the optimal investment. For simplicity, all the derivation is done by assuming that the interest and discount rate are deterministic. Nevertheless, the argument is valid for stochastic interest and discount rate as well.

Necessary Optimality Conditions At any time, installation of any infinitesimal unit of capital will create a stream of marginal profits. At optimum, the marginal profit from investing has to be lower than or equal to the cost of investing. As investment is irreversible, periods of excess capacity can be encountered in which marginal profit is strictly smaller than investment cost. The firm will invest if and only if capacity is depreciated or if investment cost decreases such that marginal profit is equal to the cost. The investment cost at $t$ discounted back to time 0 is $e^{-r t}$. Denote the marginal profit at time $t$ following the investment plan $I$ after discounting by $\nabla_{t} \Pi(I)$. Then, the necessary optimality conditions are given by

$$
\nabla_{t} \Pi(I) \leq e^{-r t} \quad \text { for all times } t \leq \hat{T}
$$


and

$$
\nabla_{t} \Pi(I)=e^{-r t} \quad \text { whenever } d I_{t}>0 .
$$

Alternatively, conditions (5) and (6) can also be interpreted as the KuhnTucker conditions for the optimality problem (1) with an inequality constraint $d I_{t} \geq 0$.

Marginal Profit The marginal investment at time $t$ first induces an immediate marginal gain of $\pi_{c}\left(X_{t}, C_{t}^{I}\right)$. As capital accumulation is irreversible, all future profits are increased marginally since installation by

$$
\pi_{c}\left(X_{s}, C_{s}^{I}\right) e^{-\delta(s-t)}, \quad \forall s \in[t, \hat{T}],
$$

where the discount factor $e^{-\delta(s-t)}$ is due to the depreciation of the current capital stocks ${ }^{3}$. Surely, this marginal gain has to be discounted by the interest rate as well to the initial date 0 . Overall, the expected marginal profit conditional on the information at time $t$ is indicated by

$$
\begin{aligned}
\nabla_{t} \Pi(I) & =\mathbb{E}\left[\int_{t}^{\hat{T}} e^{-r s} \pi_{c}\left(X_{s}, C_{s}^{I}\right) e^{-\delta(s-t)} d s \mid \mathcal{F}_{t}\right] \\
& =e^{\delta t} \mathbb{E}\left[\int_{t}^{\hat{T}} e^{-(r+\delta) s} \pi_{c}\left(X_{s}, C_{s}^{I}\right) d s \mid \mathcal{F}_{t}\right] .
\end{aligned}
$$

Remark 3.1 Assume for the moment that the firm is infinitely lived with $\hat{T}=\infty$. The first-order condition can be reformulated as

$$
\mathbb{E}\left[\int_{t}^{\infty} e^{-(r+\delta) s}\left[\pi_{c}\left(X_{s}, C_{s}^{I}\right)-(r+\delta)\right] d s \mid \mathcal{F}_{t}\right] \leq 0,
$$

after multiplying $e^{-\delta t}$ at the both sides of Inequality (5) and noting $e^{-(r+\delta) t}=$ $\int_{t}^{\infty}(r+\delta) e^{-(r+\delta) s} d s$. In the reversible case, the integrand at the right-hand side is always equal to zero, as marginal operating profit is always equal to the user cost of capital, $r+\delta$. In the irreversible case, the firm however aims to achieve the equality of marginal profit and user cost of capital in expectation on average in time. The inequality becomes strict when capacity is excess at time $t$.

\footnotetext{
${ }^{3}$ In the case of reversible investment, the effect on future profits does not occur because earlier investments can be withdrawn at any time. Thus, it is sufficient to consider the marginal gain at present time $t$ only.
} 
The Base Capacity To further proceed, a well-known approach is borrowed from inventory theory and operations research. In this way, the optimal base capacity policy is set up. Such a policy consists of computing certain base capacity $l_{t}$. This base capacity is the optimal capacity that the firm should hold if it were about to start operating at time $t$, regardless of the past capacity. In general, the policy reads as follows:

\section{Base Capacity Policy:}

If the current capacity exceeds the base capacity, keep the capacity without further investment; if the current capacity is below or equal to the base capacity, invest at once to match the base capacity!

Suppose that the firm follows the optimal investment plan: invests at some (random stopping) time $\tau_{0}$, waits for a while till $\tau_{1}>\tau_{0}$ and invests again. In this case, the first-order condition is binding at both times, namely,

$$
\nabla_{\tau_{0}} \Pi(I)=e^{-r \tau_{0}} \text { and } \nabla_{\tau_{1}} \Pi(I)=e^{-r \tau_{1}} .
$$

Multiplying both equations with $e^{-\delta \tau_{i}}, i=0,1$ respectively, and subtracting them from each other yields

$$
\mathbb{E}\left[\int_{\tau_{0}}^{\tau_{1}} e^{-(r+\delta) s} \pi_{c}\left(X_{s}, C_{s}^{I}\right) d s \mid \mathcal{F}_{\tau_{0}}\right]=\mathbb{E}\left[e^{-(r+\delta) \tau_{0}}-e^{-(r+\delta) \tau_{1}} \mid \mathcal{F}_{\tau_{0}}\right],
$$

where the conditional expectation is taken with respect to the information available at time $\tau_{0}$. The conditional expectation appears also at the righthand side because $\tau_{1}$ is generally random. Upon realizing that the difference on the right hand side can be written as $\int_{\tau_{0}}^{\tau_{1}}(r+\delta) e^{-(r+\delta) s} d s$, one arrives at

$$
\mathbb{E}\left[\int_{\tau_{0}}^{\tau_{1}} e^{-(r+\delta) s}\left[\pi_{c}\left(X_{s}, C_{s}^{I}\right)-(r+\delta)\right] d s \mid \mathcal{F}_{\tau_{0}}\right]=0 .
$$

As no investment occurs between $\tau_{0}$ and $\tau_{1}$, the capacity starts at some level $L$ at time $\tau_{0}$ and depreciates at the rate $\delta$, i.e.,

$$
C_{s}^{I}=L e^{-\delta\left(s-\tau_{0}\right)}
$$

for $s \in\left(\tau_{0}, \tau_{1}\right)$. By plugging this back into the equation above one arrives at

$$
\mathbb{E}\left[\int_{\tau_{0}}^{\tau_{1}} e^{-(r+\delta) s}\left[\pi_{c}\left(X_{s}, L e^{-\delta\left(s-\tau_{0}\right)}\right)-(r+\delta)\right] d s \mid \mathcal{F}_{\tau_{0}}\right]=0 .
$$


This equation has a unique solution for $L$, a $\mathcal{F}_{\tau_{0}}$-measurable random variablet which is denoted by $L_{\tau_{0}}^{\tau_{1}}$.

The level $L_{\tau_{0}}^{\tau_{1}}$ will be the optimal capacity at time $\tau_{0}$ if a blocked interva/5 starts at time $\tau_{0}$. In general, the firm asks at time $\tau_{0}$ : when is the next time to invest (marginally or in lumps)? It takes the whole variety of possible levels $\left(L_{\tau_{0}}^{\tau_{1}}\right)_{\tau_{1}>\tau_{0}}$ into consideration. Then, which is the proper one to take? Due to the irreversibility constraint, the lowest level is chosen as it is most favorable in the sense that it gives the maximal flexibility for future decisions. As a result,

$$
l_{\tau_{0}}=\underset{\tau_{1}>\tau_{0}}{\operatorname{essinf}} L_{\tau_{0}}^{\tau_{1}}
$$

is defined as the base capacity.

Alternatively, the definition of the base capacity as the lowest of all levels $L_{\tau_{0}}^{\tau_{1}}$ can be validated in the following way. Suppose that current capacity exceeds some $L_{\tau_{0}}^{\tau_{1}}$ and assume $\delta=0$ for simplicity. From irreversibility, it is clear that $C_{s}>L_{\tau_{0}}^{\tau_{1}}$ for all times $s \in\left(\tau_{0}, \tau_{1}\right)$. By the definition of $L_{\tau_{0}}^{\tau_{1}}$, one obtains

$$
\begin{aligned}
\mathbb{E}\left[\int_{\tau_{0}}^{\tau_{1}} e^{-r s} \pi_{c}\left(X_{s}, C_{s}\right) d s \mid \mathcal{F}_{\tau_{0}}\right] & <\mathbb{E}\left[\int_{\tau_{0}}^{\tau_{1}} e^{-r s} \pi_{c}\left(X_{s}, L_{\tau_{0}}^{\tau_{1}}\right) d s \mid \mathcal{F}_{\tau_{0}}\right] \\
& =\mathbb{E}\left[e^{-r \tau_{0}}-e^{-r \tau_{1}} \mid \mathcal{F}_{\tau_{0}}\right] .
\end{aligned}
$$

It follows that

$$
\begin{aligned}
\nabla_{\tau_{0}} \Pi(I) & =\mathbb{E}\left[\int_{\tau_{0}}^{\tau_{1}} e^{-r s} \pi_{c}\left(X_{s}, C_{s}\right) d s \mid \mathcal{F}_{\tau_{0}}\right]+\mathbb{E}\left[\int_{\tau_{1}}^{\hat{T}} e^{-r s} \pi_{c}\left(X_{s}, C_{s}\right) d s \mid \mathcal{F}_{\tau_{0}}\right] \\
& <\mathbb{E}\left[e^{-r \tau_{0}}-e^{-r \tau_{1}} \mid \mathcal{F}_{\tau_{0}}\right]+\mathbb{E}\left[\int_{\tau_{1}}^{\hat{T}} e^{-r s} \pi_{c}\left(X_{s}, C_{s}\right) d s \mid \mathcal{F}_{\tau_{0}}\right] \\
& =\mathbb{E}\left[e^{-r \tau_{0}}-e^{-r \tau_{1}} \mid \mathcal{F}_{\tau_{0}}\right]+\nabla_{\tau_{1}} \Pi(I) \leq e^{-r \tau_{0}},
\end{aligned}
$$

where the first-order constraint is used in the last line. This shows that the necessary condition for investment at time $\tau_{0}$ is that the current capacity be

\footnotetext{
${ }^{4}$ The derivation given here is heuristic. Thus, the proof is not provided for the uniqueness of the solution to this implicit equation. This argument can be made rigorous however by considering that the marginal operating profit $\pi_{c}$ is strictly decreasing to 0 in capacity.

${ }^{5}$ Please refer to the full discussion in Section 4.
} 
less than or equal to all levels $L_{\tau_{0}}^{\tau_{1}}$ for $\tau_{1}>\tau_{0}$, justifying our definition of the base capacity.

Tracking the base capacity Generally, the base capacity $l$ is a widelyfluctuating stochastic process. Irreversibility prevents the firm from exactly matching the base capacity at all times, e.g., when downward jumps occur or when the base capacity decreases at a higher rate than $\delta$ or when the base capacity decreases in a non-differentiable way as is typical for diffusion models. Therefore, a feasible capacity process $C_{t}$ has to be found out that tracks the base capacity as closely as possible.

According to the optimal investment policy (to invest when $C_{t} \leq l_{t}$, but not when $\left.C_{t}>l_{t}\right), C_{t} \geq l_{t}$ has to be always ensured at all times. Consequently, the correct means is to look for the smallest feasible capacity that dominates the base capacity.

If there is no depreciation, i.e., $\delta=0, C$ must be a nondecreasing process. Therefore, $C_{t} \geq C_{s}$ for $t>s$. Meanwhile, in accordance with the requirement $C_{s} \geq l_{s}, C_{t} \geq l_{s}$ is achieved for all $s \leq t$, or equivalently,

$$
C_{t} \geq \sup _{s \leq t} l_{s} .
$$

Being the running maximum of the base capacities, $\sup _{s \leq t} l_{s}$ is surely a nondecreasing process, and hence a feasible capacity. Therefore,

$$
C_{t}=\sup _{s \leq t} l_{s}
$$

is the smallest feasible capacity that dominates the base capacity.

For the general case $(\delta>0)$, it is better to study the process $A_{t}=C_{t} e^{\delta t}$ which is nondecreasing as required. By the same reasoning as above, it is demonstrated that $A$ has to satisfy the relationship

$$
A_{t}=\sup _{s \leq t} l_{s} e^{\delta s} .
$$

The feasible capacity becomes then

$$
C_{t}=e^{-\delta t} \sup _{s \leq t} l_{s} e^{\delta s} .
$$


The remaining question here is how to get the corresponding investment plan? Trivially, $C^{I}=I$ in the case of no depreciation. In general, one can derive the investment plan from Equation (1), or equivalently, $d I_{t}=$ $d C_{t}^{I}+\delta C_{t}^{I} d t$

Definition 3.2 For a given optional process $l$ and depreciation rate $\delta \geq 0$,

$$
C_{t}^{l, \delta}=e^{-\delta t} \sup _{s \leq t}\left(l_{s} e^{\delta s}\right)
$$

is the capacity that tracks $l$ at depreciation rate $\delta$. The investment plan that finances $C^{l, \delta}$ is denoted by $I^{l, \delta}$ and satisfies

$$
d I_{t}^{l, \delta}=d C_{t}^{l, \delta}+\delta C_{t}^{l, \delta} d t
$$

Note that the capacity that tracks the base capacity satisfies

$$
d C_{t}^{l, \delta}=-\delta C_{t}^{l, \delta} d t+e^{-\delta t} d A_{t}^{l, \delta}
$$

where $A^{l, \delta}$ is given by 10 . It follows that

$$
d I_{t}^{l, \delta}=e^{-\delta t} d A_{t}^{l, \delta}
$$

Investment takes place if and only if the process $A^{l, \delta}$ increases; this in turn happens only if the process $\left(l_{s} e^{\delta s}\right)$ reaches a new all time high.

The Backward Equation: A quick Test for Optimality Based on the analysis above, it is known that the optimal capacity tracks the base capacity. Moreover, the first-order constraint is binding if investment at some (random) time $\tau$ is optimal. In all, one has the equation

$$
\mathbb{E}\left[\int_{\tau}^{\hat{T}} e^{-(r+\delta) s} \pi_{c}\left(X_{s}, e^{-\delta s} \sup _{u \leq s} l_{u} e^{\delta u}\right) d s \mid \mathcal{F}_{t}\right]=e^{-(r+\delta) \tau}
$$

As defined in tracking the base capacity, the process $\left(l_{s} e^{\delta s}\right)$ has a new all time high at $\tau$, or simply, $l_{\tau} e^{\delta \tau} \geq l_{s} e^{\delta s} \forall, s<\tau$. Hence, it is sufficient to check the values of $\left(l_{u} e^{\delta u}\right)$ for all time $u$ after $\tau$ only during the interval $[\tau, u]$ to determine whether a new maximum is reached. It follows that the first-order condition can be rewritten as in the following lemma. 
Lemma 3.3 The base capacity satisfies the backward equation

$$
\mathbb{E}\left[\int_{\tau}^{\hat{T}} e^{-(r+\delta) s} \pi_{c}\left(X_{s}, e^{-\delta s} \sup _{\tau \leq u \leq s} l_{u} e^{\delta u}\right) d s \mid \mathcal{F}_{\tau}\right]=e^{-(r+\delta) \tau}
$$

for all (stopping) times $\tau<\hat{T}$.

As shown by Bank and ElKaroui (2002), there always exists one unique solution of the base capacity. Meanwhile, it is worth noting that this backward equation is used not just to achieve the necessary condition for optimality. Indeed, it is not only necessary but also sufficient for optimality. Once a process $l$ is obtained to solve this equation, the capacity that tracks $l$ and the corresponding investment plan are shown to be optimal.

Theorem 3.4 (Optimal Investment) Suppose that the process $l$ satisfies the Backward Equation (13). Then the optimal capacity is the one that tracks the base capacity,

$$
C^{*}=C^{l, \delta}
$$

and the optimal investment plan is $I^{*}=I^{l, \delta}$.

The rigorous proof is given in the Appendix.

Algorithmic Remarks The discussion of this section as summarized in Theorem 3.4 provides a complete solution for all irreversible investment problems. Here, we outline how to compute numerically the solution. First, the random variables $L_{\tau_{0}}^{\tau_{1}}$ is obtained by inverting implicit equations (8). The next step consists in solving the optimization problem (9) to get the base capacity. It gives immediately the optimal capacity by 11 and the optimal investment plan by $(12)$.

Remark 3.5 The same argument can be simply generalized to the case with stochastic interest and discount rate. Under this construction, the optimal investment policy is characterized as in the next theorem:

Corollary 3.6 (Optimal Investment for Stochastic Interest and Discount Rate) If the process l satisfies the Backward Equation

$\mathbb{E}\left[\int_{\tau}^{\hat{T}} e^{\int_{0}^{s}-\left(r_{v}+\delta_{v}\right) d v} \pi_{c}\left(X_{s}, e^{-\int_{0}^{s} \delta_{v} d v} \sup _{\tau \leq u \leq s} l_{u} e^{\int_{0}^{u} \delta_{v} d v}\right) d s \mid \mathcal{F}_{\tau}\right]=e^{\int_{0}^{\tau}-\left(r_{v}+\delta_{v}\right) d v}$,

then the optimal investment plan is $I^{*}=I^{l, \delta}$ which brings the optimal capacity $C^{*}=C^{l, \delta}$ tracking the base capacity. 


\subsection{A Comparison of Different Methods to Sequential Irreversible Investment Problems}

As mentioned in Introduction, Pindyck (1988) solves the sequential irreversible investment decision problems by the contingent claim option approach or the dynamic programming method. The dynamic program specified in Equation (1) is treated as a sequence of optimal stopping problem. Instead of focusing on how much to invest at each time, he starts from when the infinitesimal stock of capital should be invested. This is exactly the starting point of our method to concern the marginal effect of invest at any given time. Similarly, Bertola (1998) solves the maximization problem (1) by identifying the optimality condition in the sense of marginal effect.

On this basis, different techniques are applied to achieve the maximum, although all the methods finally come to the same threshold level for the investment. Pindyck (1988) obtains the optimal trigger level of the investment by solving the Hamilton-Jacobi-Bellman equations. Sticking to the marginal effect, Bertola (1998) identifies the marginal profit of the investment and solves its stochastic differential equation after assuming that there is a control barrier on the marginal profit. While, the method of this paper as fully developed in Section 4.2 is to search for the base capacity which tracks the threshold value of the investment. This approach aiming to identify the base capacity is based on the martingale representation method, which is first proposed by Bank and ElKaroui (2002) for singular control problems and applied to optimal consumption problems by Bank and Riedel (2001), to single irreversible investment problems by $\mathrm{Su}$ (2005) and to various stochastic optimization problems by Bank and Föllmer (2003) and the literature therein. In all, the sequential irreversible investment is by means of this method regarded as a sequence of the singular control problems of marginal investment. The optimal investment plan is characterized via a base capacity obtained by solving the backward equation (13).

Compared to the other approaches, this method has mainly three advantages. First as fully introduced in the former subsection, it incorporates economic intuition in the derivation, enabling one to better understand the irreversible investment problems. More importantly, it is well applicable to a rather general irreversible investment model and allows a general qualitative characterization of the optimal investment. In addition, this method is pow- 
erful by providing explicit solutions to some special cases where economic shocks are modelled by Lévy processes and the operating profit function is of Cobb-Douglas style, which will be more addressed later in Section 5 .

\section{Qualitative Properties of Irreversible In- vestments}

\subsection{Free and Blocked Intervals of Irreversible Invest- ments under Certainty}

In the analysis of the deterministic case, Arrow (1968) distinguishes between free and blocked intervals. In free intervals, the irreversibility constraint does not bind and investment occurs at some rate $d I_{t}=i_{t} d t$. While, the firm would like to disinvest in blocked intervals which is impossible though. Thus, $d I_{t}=0$ is obtained in blocked intervals.

The diffusion case studied by Bertola (1998), Pindyck (1988) and Boyarchenko (2004) creates such blocked intervals as well. Nevertheless, there exist no free intervals in the sense of Arrow (1968). Whenever investment occurs, it happens in a singular way: the set of time points at which investment occurs is of Lebesgue measure zero; hence there is no rate of investment. This is due to the nature of BMs and diffusions. Generally, if one wants to keep a BM below some boundary, actions have to be taken at very irregular time steps.

\subsection{Qualitative Analysis on Irreversible Investments under Uncertainty}

Given the general model discussed in the present paper, there exist in all three phenomena in irreversible investment. That is, every investment plan I can be decomposed into three parts,

$$
I=I^{a}+I^{j}+I^{\perp}
$$

where $I_{t}^{a}=\int_{0}^{t} i_{u}^{a} d u, \forall t \in[0, \hat{T}]$ is the smooth investment with an absolutely continuous plan, $I_{t}^{j}=\sum_{n: \tau_{n} \leq t} \Delta_{n}, \forall t \in[0, \hat{T}]$ consists of lump sum investments 
$\Delta_{n}$ that take place at stopping times $\left(\tau_{n}\right)_{n \geq 0}$, and $I^{\perp}$ describes the singular part of the investment plan.

Smooth Investment A random time interval $\left[\tau_{0}, \tau_{1}\right]$ is defined as a free interval when smooth investment appears. Throughout the free interval, investment occurs at a positive rate, i.e.,

$$
i_{u}^{a}>0, \quad \forall u \in\left(\tau_{0}, \tau_{1}\right) .
$$

The result of Arrow (1968) can be generalized to the irreversible investment under uncertainty: The investment rate in free intervals corresponds to reversible case in the sense of the following theorem.

Theorem 4.1 In free intervals $\left[\tau_{0}, \tau_{1}\right]$, marginal profit is equal to the user cost of capital, namely,

$$
\pi_{c}\left(X_{t}, C_{t}^{I}\right)=r+\delta \quad \text { a.s. }
$$

for all $t \in\left(\tau_{0}, \tau_{1}\right)$.

Proof: On a free interval where investment occurs continuously, the irreversibility constraint always binds as

$$
\mathbb{E}\left[\int_{t}^{\hat{T}} e^{-(r+\delta) s} \pi_{c}\left(X_{s}, C_{s}^{I}\right) d s \mid \mathcal{F}_{t}\right]=e^{-(r+\delta) t} .
$$

Define

$$
H=\int_{0}^{\hat{T}} e^{-(r+\delta) s} \pi_{c}\left(X_{s}, C_{s}^{I}\right) d s
$$

and its conditional expectation given the information at time $t$ as a martingale

$$
M_{t}=\mathbb{E}\left[H \mid \mathcal{F}_{t}\right] .
$$

It gives that in the free interval

$$
M_{t}-\int_{0}^{t} e^{-(r+\delta) s} \pi_{c}\left(X_{s}, C_{s}^{I}\right) d s=e^{-(r+\delta) t} .
$$

In particular, $M$ is a martingale with an absolutely continuous sample path. As is well known, such martingales are constant (cf. Protter (1995), Chapter 
II, pp. $64-65)^{6}$. In this way, $M_{t}=M_{0}$ for all $t$. Taking derivatives on both sides of the equation yields then

$$
\pi_{c}\left(X_{t}, C_{t}^{I}\right)=r+\delta
$$

as desired.

Example: Poisson process with figure

Lumpy Investment: Stopping Times and Information Surprises Lumpy investment $I^{j}$ takes place only at stopping times $\tau \leq \hat{T}$. Especially, we define those intervals between two stopping times during which no investment occurs as blocked intervals. Put it more explicitly, we have in a blocked interval $\left[\tau_{0}, \tau_{1}\right]$

$$
\triangle I_{\tau_{0}}, \triangle I_{\tau_{1}}>0, \text { but } \triangle I_{s}=0 \forall s \in\left(\tau_{0}, \tau_{1}\right)
$$

In contrast to the deterministic case, it is typically not the case that the firm equates marginal operating profit and the user cost of capital when it invests in blocked intervals. Instead, uncertainty usually leads to a lower capacity as shown in the following theorem and example (Please also compare it in more general Lévy examples later in Section 6).

Theorem 4.2 Suppose that the optimal investment plan has a jump at the stopping time $\tau$. Then we have

$$
\pi_{c}\left(X_{\tau}, C_{\tau}^{I}\right) \geq r+\delta
$$

In words: the capacity never jumps to an excess capacity (where the excess capacity is defined with respect to the operating profit).

Proof: Let $\tau$ be a stopping time with $\Delta I_{\tau}>0$. For shorter notation, denote the difference of the marginal operating profit and the user cost of capital by $\zeta_{t}=\pi_{c}\left(X_{t}, C_{t}^{I}\right)-(r+\delta)$. In this way, It is only necessary to show $\zeta_{\tau} \geq 0$. Fix $\varepsilon>0$. Let $\rho=\inf \left\{t \geq \tau: \zeta_{t} \geq-\varepsilon\right\}$ be the first time when $\zeta$

\footnotetext{
${ }^{6}$ If a martingale has an absolutely continuous sample path, it must have finite variation. As stated in Protter (1995), a continuous martingale with paths of finite variation is constant.
} 
is greater than or equal $-\varepsilon$. The first order conditions, $\nabla_{\tau} \Pi(I)=e^{-r \tau}$ and $\nabla_{\rho} \Pi(I) \leq e^{-r \rho}$, are equivalent to

$$
\begin{aligned}
& \mathbb{E}\left[\int_{\tau}^{\hat{T}} e^{-(r+\delta) s} \zeta_{s} d s \mid \mathcal{F}_{\tau}\right]=0 \\
& \mathbb{E}\left[\int_{\rho}^{\hat{T}} e^{-(r+\delta) s} \zeta_{s} d s \mid \mathcal{F}_{\rho}\right] \leq 0 .
\end{aligned}
$$

We obtain by taking the conditional expectation at time $\tau$ of their differences,

$$
0 \leq \mathbb{E}\left[\int_{\tau}^{\rho} e^{-(r+\delta) s} \zeta_{s} d s \mid \mathcal{F}_{\tau}\right]
$$

By the definition of $\rho$, it follows that

$$
0 \leq-\varepsilon \mathbb{E}\left[\int_{\tau}^{\rho} e^{-(r+\delta) s} d s \mid \mathcal{F}_{\tau}\right]
$$

This is only possible when $\rho=\tau$ almost surely as $\rho \geq \tau$ as defined. Therefore, we have (from right-continuity of $X$ and $C^{I}$ ) $\zeta_{\tau} \geq-\varepsilon$. As $\epsilon$ was arbitrary, $\zeta_{\tau} \geq 0$ follows.

Example 4.3 Consider a simple infinite horizon model in which there is only one shock taking place at time 1. Formally, $X_{t}=1$ for $0 \leq t<1$. At time 1, the shock jumps to either a good or a bad state with the same probability, i.e., $\mathbb{P}\left[X_{1}=\xi\right]=\mathbb{P}\left[X_{1}=\eta\right]=1 / 2$ for $\xi>1>\eta$. After time $1, X$ stays constant, that is, $X_{t}=X_{1}$, for $t>1$. Let $\left(\mathcal{F}_{t}\right)_{t \in[0, \infty]}$ be the filtration generated by $X$. The profit function is separable in the form of $\pi(x, c)=x \pi(c)$ for some nice function $\pi$. In addition, there is no depreciation, $\delta=0$.

It is easy to check that the following investment policy is optimal. The optimal base capacity at time 1 satisfies $X_{1} \pi^{\prime}\left(l_{1}\right)=r$ and stays constant afterwards, $l_{t}=l_{1}$ for $t \geq 1$. Let $h$ and $b$ be the optimal base capacities after the good and bad shock, respectively. Then, we have $\xi \pi^{\prime}(h)=r$ and $\eta \pi^{\prime}(b)=r$ with $b<h$. Between time 0 and 1 , $l$ again stays constant after time $0, l_{t}=l_{0}$. Intuitively, it is due to the fact that no new information is 
released during that interval. At time 0 , the optimal level $l_{0}$ lies between $b$ and $h$ and gives the first-order condition in equality,

$$
1=\mathbb{E} \int_{0}^{\infty} e^{-r t} X_{t} \pi^{\prime}\left(\sup _{s \leq t} l_{u}\right) d t .
$$

After time 1 , the capacity stays constant at $l_{0}$ all the time afterward with probability $1 / 2$. Otherwise, it jumps to h at time 1 when a good shock occurs. It gives then

$$
0=\frac{1}{2 r} \pi^{\prime}\left(l_{0}\right)+\frac{1}{2 r}\left[\left(1-e^{-r}\right) \pi^{\prime}\left(l_{0}\right)+e^{-r} \pi^{\prime}(h)\right]
$$

or equivalently,

$$
\pi^{\prime}\left(l_{0}\right)+\frac{e^{-r}}{2}\left(\pi^{\prime}(h)-\pi^{\prime}\left(l_{0}\right)\right)=r .
$$

As the term in bracket is negative, it is clear that

$$
\pi^{\prime}\left(l_{0}\right)>r .
$$

In blocked intervals, we have initially excess capacity in comparison with the benchmark reversible case. From the derivation of the base capacity, it is obvious that the firm tries to equate the marginal operating profit and the user cost of capital on average:

Theorem 4.4 In blocked intervals, the marginal profit equals the user cost of capital on average in expectation, formally

$$
\mathbb{E}\left[\int_{\tau_{0}}^{\tau_{1}} e^{-(r+\delta) s}\left\{\pi_{c}\left(X_{s}, C_{s}^{I}\right)-(r+\delta)\right\} d s \mid \mathcal{F}_{\tau_{0}}\right]=0 .
$$

Arrow has shown that lump sum investments do not occur except at time zero. The same holds true in the BM case analyzed by Bertola (1998) and Pindyck (1988). In general, jumps are however quite possible. To fully describe the jump effect of the investment, we introduce the concept of information surprises. As defined in Hindy and Huang (1992), information surprises include unpredictable stopping times and shocks at a predictable time with a discontinuous filtration at the predictable time. The filtration is discontinuous in the sense that $\mathcal{F}_{t-} \neq \mathcal{F}_{t}$. Consequently, there is some information surprise even if the time of a certain event is known or predictable. As given in the next theorem, the occurrence of lumpy investments is closely related to information surprises. 
Theorem 4.5 After the initial investment, jumps in investment occur only at information surprises.

PROOF: Here we will show that it is never optimal to have a jump in the investment except at information surprises. As defined in Section $2,\left(I_{t}\right)_{t \in[0, \hat{T}]}$ is a right-continuous and nondecreasing adapted process. Assume $I_{t}$ has a jump at the stopping time $\tau_{0} \in[0, \hat{T}]$. It suggests that

$$
I_{s}<I_{\tau_{0}} \quad \forall s \in\left[0, \tau_{0}\right),
$$

and that the corresponding capacity levels satisfy the following inequality

$$
C_{\tau_{0}}>C_{t} \quad \forall t \in\left(\tau_{0}-\epsilon, \tau_{0}\right)
$$

for a very small $\epsilon$ such that $\tau_{0}-\epsilon$ is sufficiently close to $\tau_{0}$.

Since investment occurs only at time $\tau_{0}$, we have

$$
\mathbb{E}\left[\int_{\tau_{0}}^{\hat{T}} e^{-(r+\delta) s}\left\{\pi_{c}\left(X_{s}, C_{s}^{I}\right)-(r+\delta)\right\} d s \mid \mathcal{F}_{\tau_{0}}\right]=0 .
$$

Meanwhile, it is always valid that for any time $t$

$$
\mathbb{E}\left[\int_{t}^{\hat{T}} e^{-(r+\delta) s}\left\{\pi_{c}\left(X_{s}, C_{s}^{I}\right)-(r+\delta)\right\} d s \mid \mathcal{F}_{t}\right] \leq 0 .
$$

As there is no information surprise at time $\tau_{0}$, we have $\mathcal{F}_{\tau_{0}-}=\mathcal{F}_{\tau}$ and hence $\mathcal{F}_{\tau_{0}-\epsilon} \subset \mathcal{F}_{\tau_{0}}$. In this way, it yields then

$$
\begin{array}{r}
\mathbb{E}\left[\int_{\tau_{0}-\epsilon}^{\hat{T}} e^{-(r+\delta) s}\left\{\pi_{c}\left(X_{s}, C_{s}^{I}\right)-(r+\delta)\right\} d s \mid \mathcal{F}_{\tau_{0}-\epsilon}\right] \leq \\
\mathbb{E}\left[\int_{\tau_{0}}^{\hat{T}} e^{-(r+\delta) s}\left\{\pi_{c}\left(X_{s}, C_{s}^{I}\right)-(r+\delta)\right\} d s \mid \mathcal{F}_{\tau_{0}-\epsilon}\right]=0,
\end{array}
$$

after taking conditional expectation given the information at time $\tau_{0}-\epsilon$. 
However, a contradictory result is obtained after reformulating the firstorder condition at $\tau_{0}-\epsilon$

$$
\begin{aligned}
\mathbb{E} & {\left[\int_{\tau_{0}}^{\hat{T}} e^{-(r+\delta) s}\left\{\pi_{c}\left(X_{s}, C_{s}^{I}\right)-(r+\delta)\right\} d s \mid \mathcal{F}_{\tau_{0}-\epsilon}\right] } \\
= & \mathbb{E}\left[\int_{\tau_{0}-\epsilon}^{\tau_{0}} e^{-(r+\delta) s}\left\{\pi_{c}\left(X_{s}, C_{s}^{I}\right)-(r+\delta)\right\} d s \mid \mathcal{F}_{\tau_{0}-\epsilon}\right] \\
& +\mathbb{E}\left[\int_{\tau_{0}}^{\hat{T}} e^{-(r+\delta) s}\left\{\pi_{c}\left(X_{s}, C_{s}^{I}\right)-(r+\delta)\right\} d s \mid \mathcal{F}_{\tau_{0}-\epsilon}\right] \\
= & \mathbb{E}\left[\int_{\tau_{0}-\epsilon}^{\tau_{0}} e^{-(r+\delta) s}\left\{\pi_{c}\left(X_{s}, C_{s}^{I}\right)-(r+\delta)\right\} d s \mid \mathcal{F}_{\tau_{0}-\epsilon}\right] .
\end{aligned}
$$

Based on the fact that $\pi_{c}(x, c)$ is decreasing in $c$, one can easily get $\pi_{c}\left(X_{\tau_{0}}, C_{\tau_{0}}^{I}\right)<\pi_{c}\left(X_{t}, C_{t}^{I}\right)$ for $t \in\left(\tau_{0}-\epsilon, \tau_{0}\right)$ as $X_{t} \approx X_{\tau}$ with the sufficiently small $\epsilon$. It hence leads to

$$
(r+\delta) \leq \pi_{c}\left(X_{\tau_{0}}, C_{\tau_{0}}^{I}\right)<\pi_{c}\left(X_{t}, C_{t}^{I}\right)
$$

for all $t \in\left(\tau_{0}-\epsilon, \tau_{0}\right)$ by combining the statement in Theorem 4.2. Clearly, it gives the result

$$
\mathbb{E}\left[\int_{\tau_{0}}^{\hat{T}} e^{-(r+\delta) s}\left\{\pi_{c}\left(X_{s}, C_{s}^{I}\right)-(r+\delta)\right\} d s \mid \mathcal{F}_{\tau_{0}-\epsilon}\right]>0,
$$

which contradicts the assertion (14). Thus, a jump in investment appears only at information surprises.

As one special case, the irreversible investment under certainty studied in Arrow (1968) possesses complete information set during the whole investment plan. As a result, lumpy investment takes place only at the initial time:

Corollary 4.6 In Arrows model, jumps occur only at time 0.

In addition, it is worthwhile to point out that there is no information surprise when the information filtration is generated by BMs. Consequently, lumpy investment appears in this case only once at the initial date.

Corollary 4.7 If the information flow is continuous (as if generated by some $B M)$, jumps in investment occur only at time 0 . 
PROOF: No information surprise means that all the stopping times are predictable and also the filtration at predictable times is always continuous. When the information filtration is generated by BMs, it is well-known that the stopping times under this construction are always predictable. Furthermore, as shown by Meyer (1963), the information structure is quasi leftcontinuous if the generated process is continuous at predictable stopping times and has the strong Markov property. In this case, the filtration is surely continuous since a BM is known a process with a continuous path and strong Markov property. It proves then the corollary.

Singular Investment Such investment are often observable whenever a diffusion or a BM is present. It is singular with respect to the short time $d t$. As the diffusion process moves continuously with infinite variations, the derived process $\left(L_{t}\right)_{t \in[0, \hat{T})}$ arrives a local minimum within any small interval. The set of disjoint times when local minima occur is countable but dense almost surely such that no one can "properly" specify those time points. Or equivalently, investment occurs at nearly adjacent dates and fluctuates in a nowhere differentiable fashion. Consequently, we cannot characterize the interval where singular investment occurs as well as its property.

\section{Comparative Statics}

An advantage of this approach to the irreversible investment theory is that it easily leads to general monotone comparative statics results. We are going to illustrate it in this section by two results. First, it is shown that the base capacity is monotonically increasing in shocks $X$ whenever the operating profit function has increasing differences in shocks and capacity. The general theory by Topkis (1978) and Milgrom and Shannon (1994) suggests that this property is necessary to have monotone comparative statics. Second, a natural result is established that the firm size is decreasing in the user cost of capital.

Assumption 5.1 The operating profit function is supermodular, that is,

$$
\frac{\partial^{2}}{\partial x \partial c} \pi(x, c) \geq 0 .
$$

Alternatively: the operating function has increasing differences (see Topkis). 
Theorem 5.2 Under Assumption 5.1, the base capacity l is monotonically increasing in $X$.

Proof: Let $X$ and $Y$ be two semi-martingales with $X \geq Y$ a.s. Denote their corresponding base capacity levels by $l^{X}$ and $l^{Y}$. As the base capacity level is the essential infimum of all candidates $L_{t}^{\tau}$ in (9), it is enough to show that the $L_{t}^{\tau}$ corresponding to the exogenous stock $X$ is larger than that to $Y$, or equivalently, $L_{t}^{X, \tau}>L_{t}^{Y, \tau}$.

Define the left-hand side equation of the first order condition (8) as a function $f(L)$, i.e.,

$$
\begin{aligned}
f(L) & =\mathbb{E}\left[\int_{t}^{\tau} e^{-(r+\delta) s}\left[\pi_{c}\left(X_{s}, L e^{-\delta(s-t)}\right)-(r+\delta)\right] d s \mid \mathcal{F}_{t}\right] \\
& =\mathbb{E}\left[\int_{t}^{\tau} e^{-(r+\delta) s} \pi_{c}\left(X_{s}, L e^{-\delta(s-t)}\right) d s \mid \mathcal{F}_{t}\right]+\mathbb{E}\left[e^{-(r+\delta) \tau}-e^{-(r+\delta) t} \mid \mathcal{F}_{t}\right] .
\end{aligned}
$$

Based on the definition, $L_{t}^{\tau}$ is the unique solution of the first order condition (8). Thus, we get an equality of the two conditions subject to different shocks $X$ and $Y$ as follows:

$$
\begin{aligned}
0 & =\mathbb{E}\left[\int_{t}^{\tau} e^{-(r+\delta) s}\left[\pi_{c}\left(X_{s}, L_{t}^{X, \tau} e^{-\delta(s-t)}\right)-(r+\delta)\right] d s \mid \mathcal{F}_{t}\right] \\
& =\mathbb{E}\left[\int_{t}^{\tau} e^{-(r+\delta) s}\left[\pi_{c}\left(Y_{s}, L_{t}^{Y, \tau} e^{-\delta(s-t)}\right)-(r+\delta)\right] d s \mid \mathcal{F}_{t}\right] .
\end{aligned}
$$

As the function $f(L)$ is decreasing in $L$, it is enough to show the following inequality

$$
\begin{gathered}
\mathbb{E}\left[\int_{t}^{\tau} e^{-(r+\delta) s}\left[\pi_{c}\left(X_{s}, L e^{-\delta(s-t)}\right)-(r+\delta)\right] d s \mid \mathcal{F}_{t}\right] \geq \\
\mathbb{E}\left[\int_{t}^{\tau} e^{-(r+\delta) s}\left[\pi_{c}\left(Y_{s}, L e^{-\delta(s-t)}\right)-(r+\delta)\right] d s \mid \mathcal{F}_{t}\right] .
\end{gathered}
$$

for any a stopping time $\tau>t$. It follows immediately from the fact that $\pi_{c}$ is increasing in $x$ as $\pi$ is supermodular.

Remark 5.3 An alternative proof via Topkis (1978) is also possible. Moreover, Theorem 10 in Milgrom and Shannon (1994) suggests that supermodularity is necessary for this type of monotone comparative statics. 
Theorem 5.4 The base capacity is decreasing in the user cost of capital $r+\delta$.

Proof: First again on the basis of (9), it is sufficient to check the relationship of the user cost of capital and $L_{t}^{\tau}$. As $L_{t}^{\tau}$ is the unique solution of the first order condition, we have always $f\left(L_{t}^{\tau}\right)=0$ where $f(L)$ is as defined in the proof of Theorem 5.2. Moreover, an implicit function exists to characterize $L_{t}^{\tau}$ as a function of the user cost of capital, $L_{t}^{\tau}=L(x, r+\delta)$, if $f(L)$ is differentiable. One necessary condition to guarantee the differentiability is $\mathbb{E}\left[e^{-(r+\delta) s} \pi_{c}\left(X_{s}, L_{s} e^{-\delta(s-t)}\right) d s \mid \mathcal{F}_{t}\right]<\infty \forall L>0$, which is always satisfied since $\pi(x, c)$ is concave with $\pi_{c}(x, c)<\infty$ for $c \in(0, \infty]^{7}$.

By applying the implicit function theorem, the effect of a change in $(r+\delta)$ on $L_{t}^{\tau}$ can be characterized in the following way:

$$
\frac{\partial L_{t}^{\tau}}{\partial(r+\delta)}=-\frac{\partial f\left(L_{t}^{\tau}\right) / \partial(r+\delta)}{\partial f\left(L_{t}^{\tau}\right) / \partial L_{t}^{\tau}}
$$

The denominator is easily obtained as

$$
\frac{\partial f\left(L_{t}^{\tau}\right)}{\partial L_{t}^{\tau}}=\mathbb{E}\left[\int_{t}^{\tau} e^{-(r+\delta) s} \pi_{c c}\left(X_{s}, L_{t}^{\tau} e^{-\delta(s-t)}\right) e^{-\delta(s-t)} d s \mid \mathcal{F}_{t}\right]
$$

which is always negative since $\pi$ is convex in capacity.

\footnotetext{
${ }^{7}$ Here, $L=0$ is not concerned since it is never the unique solution to the first order condition $\mathbb{E}\left[\int_{t}^{\tau} e^{-(r+\delta) s} \pi_{c}\left(X_{s}, L e^{-\delta(s-t)}\right) d s \mid \mathcal{F}_{t}\right]=\mathbb{E}\left[e^{-(r+\delta) t}-e^{-(r+\delta) \tau} \mid \mathcal{F}_{t}\right]<\infty$ for $t<\tau$.
} 
Meanwhile, the nominator is calculated and further reduced by using the condition $f\left(L_{t}^{\tau}\right)=0$ :

$$
\begin{aligned}
& \frac{\partial f\left(L_{t}^{\tau}\right)}{\partial(r+\delta)} \\
= & \mathbb{E}\left[\int_{t}^{\tau}-s e^{-(r+\delta) s} \pi_{c}\left(X_{s}, L_{t}^{\tau} e^{-\delta(s-t)}\right) d s \mid \mathcal{F}_{t}\right]-\mathbb{E}\left[\tau e^{-(r+\delta) \tau}-t e^{-(r+\delta) t} \mid \mathcal{F}_{t}\right] \\
= & \mathbb{E}\left[\int_{t}^{\tau}-s e^{-(r+\delta) s} \pi_{c}\left(X_{s}, L_{t}^{\tau} e^{-\delta(s-t)}\right) d s \mid \mathcal{F}_{t}\right]-\mathbb{E}\left[\tau e^{-(r+\delta) \tau}-t e^{-(r+\delta) t} \mid \mathcal{F}_{t}\right] \\
< & \mathbb{E}\left[\int_{t}^{\tau}-s e^{-(r+\delta) s} \pi_{c}\left(X_{s}, L_{t}^{\tau} e^{-\delta(s-t)}\right) d s \mid \mathcal{F}_{t}\right]-t \mathbb{E}\left[e^{-(r+\delta) \tau}-e^{-(r+\delta) t} \mid \mathcal{F}_{t}\right] \\
= & \mathbb{E}\left[\int_{t}^{\tau}-s e^{-(r+\delta) s} \pi_{c}\left(X_{s}, L_{t}^{\tau} e^{-\delta(s-t)}\right) d s \mid \mathcal{F}_{t}\right]+t \mathbb{E}\left[\int_{t}^{\tau} e^{-(r+\delta) s} \pi_{c}\left(X_{s}, L_{t}^{\tau} e^{-\delta(s-t)}\right) d s \mid \mathcal{F}_{t}\right] \\
= & -\mathbb{E}\left[\int_{t}^{\tau}(s-t) e^{-(r+\delta) s} \pi_{c}\left(X_{s}, L_{t}^{\tau} e^{-\delta(s-t)}\right) d s \mid \mathcal{F}_{t}\right]<0 .
\end{aligned}
$$

In this way, we have $\frac{\partial L_{t}^{\tau}}{\partial(r+\delta)}<0$, namely a decreasing relationship of the base capacity and the user cost of capital.

\section{Explicit Solutions for Lévy Shocks and a Cobb-Douglas Operating Profit Function}

Generally, numerical methods have to be adopted to identify the solutions. Nevertheless, a closed-form solution can be obtained for a infinite-lived firm $(\hat{T}=\infty)$ when economic shocks are characterized by Lévy processes and the firm is endowed with the operating profit function of the form

$$
\pi\left(X_{t}, C_{t}\right)=\frac{1}{1-\alpha} X_{t}^{\alpha} C_{t}^{1-\alpha}, \quad 0<\alpha \leq 1 .
$$

This construction is consistent with a competitive firm who produces at decreasing returns to scale or with a monopolist firm facing with a constant returns to scale production and a constant elasticity demand function as shown by Abel and Eberly (1996) and Morellec (2001). Clearly, this function is concave with the first derivative $\pi_{C}=X_{t}^{\alpha} C_{t}^{-\alpha}$. In particular, the economic shocks $X_{t}$ is modelled in this paper by

$$
X_{t}=x_{0} e^{Y_{t}}
$$


where $x_{0}$ is the initial value at $t=0$ and $Y_{t}$ is a Lévy process with zero initial value. Moreover, the interest and discount rate are assumed to be constant over time.

As introduced in Section 3, the irreversible investment decision problem is solved by calculating the first-order condition and solving the achieved backward equation (13). Under this construction, it is written as

$$
\mathbb{E}\left[\int_{\tau}^{\infty} e^{-(r+\delta) s} X_{s}^{\alpha}\left(e^{-\delta s} \sup _{\tau \leq u \leq s} l_{u} e^{\delta u}\right)^{-\alpha} d s \mid \mathcal{F}_{\tau}\right]=e^{-(r+\delta) \tau},
$$

which can be explicitly solved by means of the strong Markov property and time homogeneity of Lévy processes, as given in the following theorem.

Theorem 6.1 When the production function is of form (15) and economic shocks are characterized by Lévy processes, the base capacity is identified as $l_{t}=\kappa X_{t}$ with

$$
\kappa=\left(\frac{1}{r+\delta} \mathbb{E}\left[e^{\alpha\left(G_{\tau(r+\delta)}-\underline{G}_{\tau(r+\delta)}\right)}\right]\right)^{\frac{1}{\alpha}},
$$

where $G_{t}=Y_{t}+\delta t, \underline{G}_{t}$ is defined as $\underline{G}_{t}=\inf _{0 \leq u \leq t} G_{u}$ and $\tau(r+\delta)$ is an exponential distributed time with the parameter $r+\delta$.

Proof: To proof it, first construct the base capacity in form of $l_{u}=\kappa X_{u}$. Then the left hand side of Equation (16) can be reduced into

$$
\begin{aligned}
& \mathbb{E}\left[\int_{\tau}^{\infty} e^{-(r+\delta) s} X_{s}^{\alpha}\left(e^{-\delta s} \sup _{\tau \leq u \leq s} l_{u} e^{\delta u}\right)^{-\alpha} d s \mid \mathcal{F}_{\tau}\right] \\
= & \mathbb{E}\left[\int_{\tau}^{\infty} e^{-(r+\delta) s} X_{s}^{\alpha} \inf _{\tau \leq u \leq s}\left(\kappa X_{u}\right)^{-\alpha} e^{-\alpha \delta(u-s)} d s \mid \mathcal{F}_{\tau}\right] \\
= & \mathbb{E}\left[\int_{\tau}^{\infty} e^{-(r+\delta) s} \kappa^{-\alpha} \inf _{\tau \leq u \leq s}\left(\frac{x_{0} e^{Y_{s}}}{x_{0} e^{Y_{u}}}\right)^{\alpha} e^{-\alpha \delta(u-s)} d s \mid \mathcal{F}_{\tau}\right] \\
= & \mathbb{E}\left[\int_{\tau}^{\infty} e^{-(r+\delta) s} \kappa^{-\alpha} \inf _{\tau \leq u \leq s} e^{\alpha\left[\left(Y_{s}-Y_{u}\right)+\delta(s-u)\right]} d s \mid \mathcal{F}_{\tau}\right] \\
= & \mathbb{E}\left[\int_{0}^{\infty} e^{-(r+\delta)(t+\tau)} \kappa^{-\alpha} \inf _{0 \leq u \leq t} e^{\alpha\left[\left(Y_{t+\tau}-Y_{u+\tau}\right)+\delta(t-u)\right]} d t \mid \mathcal{F}_{\tau}\right],
\end{aligned}
$$


where the last step is obtained by assuming $t=s-\tau$.

It can be further simplified by the property of Lévy processes that $Y_{t}-Y_{u}$ has the same distribution as $Y_{t-u}$ and is independent of the $\sigma$-Field $\mathcal{F}_{u}$.

$$
\begin{aligned}
& E\left[\int_{0}^{\infty} e^{-(r+\delta)(t+\tau)} \kappa^{-\alpha} \inf _{0 \leq u \leq t} e^{\alpha\left[\left(Y_{t}-Y_{u}\right)+\delta(t-u)\right]} d t\right] \\
= & e^{-(r+\delta) \tau} \kappa^{-\alpha} E\left[\int_{0}^{\infty} e^{-(r+\delta) t} \inf _{0 \leq u \leq t} e^{\alpha\left(G_{t}-G_{u}\right)} d t\right]
\end{aligned}
$$

where $G_{t}=Y_{t}+\delta t$ is clearly also a Lévy process.

In this way, $\kappa$ is obtained as follows by defining $\underline{G}_{t}=\inf _{0 \leq u \leq t} G_{u}$

$$
\begin{aligned}
\kappa & =\left(\mathbb{E}\left[\int_{0}^{\infty} e^{-(r+\delta) t} \inf _{0 \leq u \leq t} e^{\alpha\left(G_{t}-G_{u}\right)} d t\right]\right)^{\frac{1}{\alpha}} \\
& =\left(\mathbb{E}\left[\int_{0}^{\infty} e^{-(r+\delta) t} e^{\alpha\left(G_{t}-\underline{G}_{t}\right)} d t\right]\right)^{\frac{1}{\alpha}} \\
& =\left(\frac{1}{r+\delta} \mathbb{E}\left[e^{\alpha\left(G_{\tau(r+\delta)}-\underline{G}_{\tau(r+\delta)}\right)}\right]\right)^{\frac{1}{\alpha}},
\end{aligned}
$$

where $\tau(r+\delta)$ is an exponential distributed time with the parameter $r+\delta$.

Example 6.2 I want to have here:

1. compound Poisson model: $Y_{t}=\sum_{n=1}^{N_{t}} Z_{n}$ for $\left(Z_{n}\right)$ iid with $\mathbb{P}\left[Z_{1}=1\right]=$ $1 / 2=\mathbb{P}\left[Z_{n}=-1\right]$ and $N$ a Poisson process. Here, we are going to have free intervals and jumps, no singular investment.

2. geometric Brownian motion

Pictures!!!!

Computation of the threshold $\kappa$ Equation (17) can be further solved in closed form. Before showing the result, let us first review two properties of Lévy processes as given in Bertoin (1996). 
Lemma 6.3 If $\tau(q)$ is an exponential distributed time with the parameter $q$ independent of the Lévy process $G$, one has

- Wiener-Hopf factorization

$$
\mathbb{E}\left[e^{z \bar{G}_{\tau(q)}}\right] \mathbb{E}\left[e^{z\left(G_{\tau(q)}-\bar{G}_{\tau(q)}\right)}\right]=\frac{q}{q-\Psi(z)},
$$

where $\bar{G}_{\tau}=\sup _{0 \leq u \leq t} G_{u}$ and $\Psi(z)$ is the the Lévy-Laplace exponent of $G$ obtained by

$$
E\left[e^{z G_{t}}\right]=e^{t \Psi(z)} .
$$

- When $G$ has no positive jumps, $\bar{G}_{\tau(q)}$ is exponentially distributed with the parameter $\Phi^{+}(q)$, the positive root of the characteristic equation $\Psi(z)=q$.

With these techniques, $\kappa$ can be solved explicitly as in the following theorem:

Theorem 6.4 $\mathbb{E}\left[e^{\alpha\left(G_{\tau(r+\delta)}-\underline{G}_{\tau(r+\delta)}\right)}\right]$ in Equation 17 is specified as

- $\left(\mathbb{E}\left[e^{\alpha\left(G_{\tau(r+\delta)}^{\prime}-\overline{G^{\prime}} \tau(r+\delta)\right)}\right]\right)^{-1}$, the inversion of the right Wiener-Hopf factor of $G_{t \in[0, \infty]}^{\prime}=-G_{t \in[0, \infty]}$ which is readily recognized for Geometric Brownian Motions (GBM) and Lévy processes of exponential type.

- $\frac{\Phi^{+}(r+\delta)(r+\delta-\Psi(\alpha))}{(r+\delta)\left(\Phi^{+}(r+\delta)-\alpha\right)}$ for Lévy processes $G_{t \in[0, \infty]}$ with only positive jumps.

Proof: It is well known that $G_{t}-\underline{G}_{t}$ and $\bar{G}_{t}-G_{t}, \forall t \in[0, \infty]$ are reflected processes at the supremum and infimum, respectively. Moreover, $G_{t}-\underline{G}_{t}$ is the dual process of $\bar{G}_{t}-G_{t}$, i.e.,

$$
G_{t}-\underline{G}_{t}=\overline{G^{\prime}} t-G_{t}^{\prime}
$$

where $G_{t \in[0, \infty]}^{\prime}=-G_{t \in[0, \infty]}$. Hence,

$$
\left.\mathbb{E}\left[e^{\alpha\left(G_{\tau(r+\delta)}-\underline{G}_{\tau(r+\delta)}\right)}\right]=\mathbb{E}\left[e^{\alpha\left(\overline{G^{\prime}} \tau(r+\delta)\right.}-G_{\tau(r+\delta)}^{\prime}\right)\right],
$$

which is the inversion of the right Wiener-Hopf factor of the lévy process $G^{\prime}$. It is readily recognized for GBMs and regular Lévy processes of exponential 
type (A general solution form is derived in Boyarchenko and Levendorskii (2002).).

For a Lévy process $\left(Y_{t}\right)_{t \in[0, \infty]}$ with only positive jumps, the corresponding $G^{\prime}$ has no positive jumps. Its supremum process evaluated at the exponentially distributed random time has a unique distribution and hence

$$
E\left[e^{\alpha \overline{G^{\prime}} \tau(r+\delta)}\right]=\frac{\Phi^{+}(r+\delta)}{\Phi^{+}(r+\delta)-\alpha},
$$

where $\Phi^{+}(r+\delta)$ is the positive root of the characteristic equation $\Psi(z)=r+\delta$ of the Lévy process $G^{\prime}$. In this way,

$$
\begin{aligned}
E\left[e^{\alpha\left(G_{\tau(r+\delta)}-\underline{G}_{\tau(r+\delta)}\right)}\right] & =\left(\mathbb{E}\left[e^{\alpha\left(G_{\tau(r+\delta)}^{\prime}-\bar{G}_{\tau(r+\delta)}\right)}\right]\right)^{-1} \\
& =E\left[e^{\left.\alpha \bar{G}_{\tau(r+\delta)}\right] / \frac{r+\delta}{r+\delta-\Psi(\alpha)}}\right. \\
& =\frac{\Phi^{+}(r+\delta)(r+\delta-\Psi(\alpha))}{(r+\delta)\left(\Phi^{+}(r+\delta)-\alpha\right)},
\end{aligned}
$$

where $\Psi(\alpha)$ is the Lévy-Laplace exponent of $G^{\prime}$.

\section{Conclusion}

This paper studies sequential irreversible investment decision problems under uncertainty. To better develop the sequential irreversible investment theory, a very general model is set up which includes all existing models discussed in the literature. Given the general model formulation, this paper first derives the existence and uniqueness theorem, which is not yet available in the literature, by providing sufficient conditions for one unique optimal investment policy with finite and infinite horizon, respectively.

Then, the optimal investment rule is derived in a very detailed and intuitive way by a new method. By means of this method, the sequential irreversible investment problem is treated as a sequence of singular control problems. As the starting point, the relationship of the marginal profit and cost of investment is first checked to achieve the necessary and sufficient 
optimality conditions. In principal, the marginal profit by installing any infinitesimal unit of capital has to be lower than or equal to the cost. Then, the marginal investment problem is solved by constructing and tracking the base capacity and calculating the resulted stochastic backward equation. After identifying the base capacity, the optimal investment policy is determined: if and only if the current capacity is lower or equal to the base capacity, investment is undertaken at once to increase the capacity to the base capacity; otherwise just keep the current capacity.

This method is advantageous mainly at the follows four aspects. First, it applies well to the model which is more general than all those studied before and includes the standard finance model where the uncertainty is specified by a semi-martingale process. In addition, it incorporates an economic interpretation in the derivation and allows a general qualitative characterization of the optimal investment. Generally, the investment plan can be characterized by three different phenomena: smooth continuous investment, lump sum investment and singular investment. The marginal profit is equal to the user cost of capital only in the free interval where the smooth investment occurs at positive rate. Lumpy sum investment is possible only with information surprises in the blocked interval. In this interval, the equality of the marginal profit and the user cost of capital is maintained only in expectation in average over time. Singular investment takes place without rate of investments whenever the uncertainty is (partly) modelled by Brownian motions. Third, this new method gives some general comparative statics results: When the operating profit function is supermodular, the base capacity increases monotonically with the exogenous shock; and the firm size always declines with the user cost of capital. Finally, explicit solutions can be obtained for a special (simple but practical) case where the firm is endowed with the operating profit function of Cobb-Douglas style and multiplicative economic shocks are modelled by Lévy processes. In this context, the base capacity is identified as multiplicative shocks multiplied by a factor $\kappa$. 


\section{References}

Abel, A. B., And J. C. Eberly (1996): "Optimal Investment with Costly Reversibility," Review of Economic Studies, 63, 581-593.

Arrow, K., And A. C. Fisher (1974): "Environmental preservation, uncertainty and irreversibility," Quarterly Journal of Economics, 88, 312319.

Arrow, K. J. (1968): "Optimal Capital Policy with Irreversible Investment," in Value, Capital, and Growth, Essays in Honor of Sir John Hicks, ed. by J. N. Wolfe, pp. 1-19. Edinburgh University Press, Edinburgh.

BALDER, E. (1989): "Infinite-dimensional Extension of a Theorem of Komlos," Probability Theory and Related Fields, 81(2), 185-188.

Bank, P., And N. ElKaroui (2002): "A Stochastic Representation Theorem with Applications to Optimization and Obstacle Problems," Annals of Probability, 32(1b), 1030-1067.

Bank, P., And H. Föllmer (2003): "American Options, Multi-Armed Bandits, and Optimal Consumption Plans: A Unified View," in ParisPrinceton Lectures in Financial Mathematics, p. to appear. Springer, New York, Heidelberg.

Bank, P., And F. Riedel (2001): "Optimal Consumption Choice Under Uncertainty with Intertemporal Substitution," Annals of Applied Probability, 11, 750-788.

Bertoin, J. (1996): Lévy Processes. Cambridge University Press.

Bertola, G. (1989): "Irreversible Investment," Working Paper, Princeton University.

(1998): "Irreversible Investment," Research in Economics, 52, 3-37.

Boyarchenko, S. (2004): "Irreversible Decisions and Record Setting News Principles," American Economic Review, 94(3), 557-568.

Boyarchenko, S., And S. Levendorskil (2002): Non-Gaussian MertonBlack-Scholes theory. New Jersey-London-Singapore-Hong Kong: World Scientific. 
Dixit, A., And R. Pindyck (1994): Investment under Uncertainty. Princeton University Press, Princeton.

Guo, X., J. Miao, and E. Morellec (2005): "Irreversible Investment with Regime Shifts," Journal of Economic Theory, 122, 37-59.

Henry, C. (1974): "Investment Decisions under Uncertainty: the Irreversibility Effect," American Economic Review, 64, 89-104.

Hindy, A., and C.-F. Huang (1992): "Intertemporal Preferences for Uncertain Consumption: a Continuous-Time Approach," Econometrica, 60, 781-801.

Jorgenson, D. W. (1963): "Capital Theory and Investment Behavior," American Economic Review, 53, 247-257.

Kabanov, Y. (1999): "Hedging and Liquidation under Transaction Costs in Currency Markets," Finance and Stochastics, 3, 237-248.

Kobila, T. (1993): "A Class of Solvable Stochastic Investment Problems Involving Singular Controls," Stochastics, 43, 29-63.

Komlós, J. (1967): "A Generalization of a Problem of Steinhaus," Acta Math. Acad. Sci. Hung, 18, 217-229.

Meyer, P. (1963): "Decomposition of Supermartingales: The Uniqueness Theorem," Illinois Journal of Mathematics, 7, 1-17.

Milgrom, P., and C. Shannon (1994): "Monotone Comparative Statics," Econometrica, 62(1), 157-180.

Morellec, E. (2001): "Asset Liquidity, Capital Structure and Secured Debt," Journal of Financial Economics, 61(173-206).

PINDYCK, R. S. (1988): "Irreversible Investment, Capacity Choice, and the Value of the Firm," American Economic Review, 79, 969-985.

Protter, P. (1995): Stochastic Integration and Differential Equations. Springer, 3rd edn.

Su, X. (2005): "A New Approach on Real Options," Working Paper, Bonn University. 
Topkis, D. (1978): "Minimizing a Submodular Function on a Lattice," Operations Research, 26, 305-321. 


\section{A Proof of the Existence and Uniqueness}

\section{A.1 Finite Horizon}

Theorem A.1 Under Assumption 2.4, there exists a unique optimal investment plan $I^{*}$.

Proof: For simplicity, assume in the proof that the interest and depreciation rate $r$ and $\delta$ are positive constants. The argument goes through also in the case of bounded, nonnegative processes with the corresponding and obvious changes, which is easily done without any difficulties but in terms of clumsier formula.

First, uniqueness follows directly from strict concavity as usual. Hence, it is not necessary to be more addressed here. The existence proof is not that trivial and consists of three steps. First, Assumption 2.4 (i) is shown to guarantee the finiteness of $\Pi(I)$. Step 2 demonstrates that one can restrict attention to those investment plans $I$ which lead to the capacities that satisfy $\mathbb{E}\left[C_{\hat{T}}^{I}\right] \leq K$, where the constant $K$ is as defined in Assumption 2.4 (ii). In the third step, a suitable variant of Komlos' Theorem (Komlós (1967), see also Balder (1989) and Kabanov (1999)) is applied to obtain a sequence of investment plans $\left(I^{n}\right)$ that converges in the Cesaro sense almost surely to some invest plan $I^{*}$. Concavity of the profit functional ensures the optimality of $I^{*}$.

Step 1. From Equation (1), one can write $d I_{t}=d C_{t}^{I}+\delta C_{t}^{I} d t$. This yields

$$
\int_{0}^{\hat{T}} e^{-r t} d I_{t}=\int_{0}^{\hat{T}} e^{-r t} d C_{t}^{I}+\int_{0}^{\hat{T}} \delta e^{-r t} C_{t}^{I} d t .
$$

Integration by parts gives

$$
\int_{0}^{\hat{T}} e^{-r t} d C_{t}^{I}=e^{-r \hat{T}} C_{\hat{T}}^{I}+\int_{0}^{\hat{T}} r e^{-r t} C_{t}^{I} d t
$$

and hence

$$
\int_{0}^{\hat{T}} e^{-r t} d C_{t}^{I}=e^{-r \hat{T}} C_{\hat{T}}^{I}+\int_{0}^{\hat{T}}(r+\delta) e^{-r t} C_{t}^{I} d t
$$


It follows then

$$
\begin{aligned}
\int_{0}^{\hat{T}} e^{-r t}\left(\pi\left(X_{t}, C_{t}^{I}\right) d t-d I_{t}\right) & \leq \int_{0}^{\hat{T}} e^{-r t}\left(\pi\left(X_{t}, C_{t}^{I}\right)-(r+\delta) C_{t}^{I}\right) d t \\
& \leq \int_{0}^{\hat{T}} e^{-r t} \pi^{*}\left(X_{t}, r, \delta\right) d t
\end{aligned}
$$

This implies consequently

$$
\Pi(I) \leq \mathbb{E} \int_{0}^{\hat{T}} e^{-r t} \pi^{*}(X(t), r, \delta) d t<\infty,
$$

and the problem has always a finite value $v^{\star}=\sup _{I} \Pi(I)<\infty$.

Step 2. In this step, an investment plan $\hat{I}$ with the corresponding capacity $\hat{C}$ is constructed such that it gives an upper bound for all reasonable plans in the sense that it is not worthwhile to have a higher capacity than $\hat{C}$. The basic idea is that it does not make sense to have a capacity higher than that one would have in the reversible case, $c^{*}$. A complication arises from the fact that $c^{*}\left(X_{s}, r, \delta\right)$ will generally be a process of unbounded variation and thus may not be a feasible capacity.

The trick here is to construct the investment plan that leads to a capacity $\hat{C} \geq c^{*}$ in a minimal way. Set

$$
\hat{C}_{t}=e^{-\delta t} \sup _{s \leq t}\left(c_{s}^{*} e^{\delta s}\right)
$$

where the notation is slightly abused by writing

$$
c_{s}^{*}=c^{*}\left(X_{s}, r, \delta\right) .
$$

Because of Assumption 2.4 and $\delta \geq 0, \hat{C}_{\hat{T}}$ is integrable as

$$
\mathbb{E}\left[\hat{C}_{\hat{T}}\right]=\mathbb{E}\left[\sup _{s \leq \hat{T}} c_{s}^{*} e^{-\delta(\hat{T}-s)}\right] \leq \mathbb{E}\left[\sup _{s \leq \hat{T}} c_{s}^{*}\right]<\infty
$$

where $\mathbb{E}\left[\sup _{s \leq \hat{T}} c_{s}^{*}\right]$ is obviously equal to $K$ specified in Assumption 2.4 with the deterministic $r$ and $\delta$. 
The investment plan

$$
\hat{I}_{t}=\hat{C}_{t}+\int_{0}^{t} \delta \hat{C}_{s} d s
$$

is the feasible plan that leads to the capacity $\hat{C}$.

The claim to be demonstrated is that one can restrict attention to plans $I$ with capacity $C^{I} \leq \hat{C}$. Let $I$ be given and write $C=C^{I}$ for shorter notation. Define $\bar{C}_{t}=\min \left\{C_{t}, \hat{C}_{t}\right\}$ and $\bar{A}_{t}=e^{\delta t} \bar{C}_{t}$. Note that $\left(A_{t}\right)_{t \in[0, \hat{T}]}$ is also nondecreasing as $\left(C_{t}\right)_{t \in[0, \hat{T}]}$. The corresponding investment plan with capacity $C^{\bar{I}}=\bar{C}$ is denoted as $\bar{I}_{t}=\int_{0}^{t} e^{\delta s} d \bar{A}_{s}$.

Under this construction, the claim is valid if $\bar{I}$ is shown to be at least as good as $I$. Taking integration by parts again yields

$$
\begin{aligned}
\Pi(\bar{I})-\Pi(I)= & \mathbb{E}\left[\int_{0}^{\hat{T}} e^{-r t}\left(\pi\left(X_{t}, \bar{C}_{t}\right)-(r+\delta) \bar{C}_{t}\right) d t\right]-\mathbb{E}\left[e^{-r \hat{T}} \bar{C}_{\hat{T}}\right] \\
& -\mathbb{E}\left[\int_{0}^{\hat{T}} e^{-r t}\left(\pi\left(X_{t}, C_{t}\right)-(r+\delta) C_{t}\right) d t\right]+\mathbb{E}\left[e^{-r \hat{T}} C_{\hat{T}}\right] \\
= & \mathbb{E}\left[\int_{0}^{\hat{T}} e^{-r t}\left[\left(\pi\left(X_{t}, \bar{C}_{t}\right)-(r+\delta) \bar{C}_{t}\right)-\left(\pi\left(X_{t}, C_{t}\right)-(r+\delta) C_{t}\right)\right] d t\right] \\
& -\mathbb{E}\left[e^{-r \hat{T}}\left(\bar{C}_{\hat{T}}-C_{\hat{T}}\right)\right]
\end{aligned}
$$

The last term is nonnegative because $\bar{C} \leq C$. The integrand in the first term is either zero when $\bar{C}=C$; or nonnegative when $\bar{C}<C$. In the second case of $\bar{C}<C$, it is clear that $C_{t}>\bar{C}_{t} \geq c_{t}^{*}$. As $C_{t}$ is located at the right of the maximum $c^{*}$ and the function $c \mapsto \pi(x, c)-(r+\delta) c$ is concave, one can find out that

$$
\pi\left(X_{t}, \bar{C}_{t}\right)-(r+\delta) \bar{C}_{t}>\pi\left(X_{t}, C_{t}\right)-(r+\delta) C_{t}
$$

These arguments altogether lead to $\Pi(\bar{I}) \geq \Pi(I)$ as desired.

Step 3. In this way, the auxiliary problem

$$
\sup _{I: C^{I} \leq \hat{C}} \Pi(I)=v^{*}
$$


has the same value as the original problem. Choose an optimal sequence $\left(I^{n}\right)$ for this auxiliary problem. Its value at time $\hat{T}$ has the following property:

$$
I_{\hat{T}}^{n}=C_{\hat{T}}^{I^{n}}+\delta \int_{0}^{\hat{T}} C_{s}^{I^{n}} d s \leq(1+\delta \hat{T}) C_{\hat{T}}^{I^{n}} \leq(1+\delta \hat{T}) \hat{C}_{\hat{T}}
$$

This suggests that

$$
\sup _{n} \mathbb{E}\left[I_{\hat{T}}^{n}\right]<\infty
$$

With this condition, Komlos Theorem (in the variant of Kabanov (1999)) can be thus applied here: assume without loss of generality that $\left(I^{n}\right)$ converges in the Cesaro sense almost surely to some $I^{*}$, that is

$$
J_{t}^{n} \triangleq \lim _{n \rightarrow \infty} \frac{1}{n} \sum_{k=1}^{n} I_{t}^{k}=I_{t}^{*} \quad \text { a.s. }
$$

Through linearity, the corresponding capacities $C^{k}$ converge also in the Cesaro sense almost surely. Moreover, the concavity of the profit function in capacity yields the final result that

$$
\Pi\left(I^{*}\right) \geq \limsup \Pi\left(J^{n}\right)=v^{*}
$$

Therefore, $I^{*}$ is the optimal investment plan that maximizes the firm's net profit.

\section{A.2 Existence for the Infinite Horizon Case}

Of course, the naive generalization of Assumption 2.4 with

$$
\mathbb{E}\left[\sup _{t<\infty} c_{t}^{*}\right]<\infty
$$

is sufficient (by repeating the proof above for the finite horizon case). However, it is too strong in the infinite horizon case because the running maximum up to infinity will be in many contexts infinity. Indeed, the following weaker version of Assumption 2.4 is sufficient to guarantee the existence of the optimal sequential investment plan with the infinite horizon.

Assumption A.2 (i) $\mathbb{E}\left[\int_{0}^{\infty} e^{-\int_{0}^{t} r_{s} d s} \pi^{*}\left(X_{t}, r_{t}, \delta_{t}\right) d t\right]<\infty \forall t \in[0, \hat{T}]$. 
(ii) $K \triangleq \mathbb{E}\left[\int_{0}^{\infty} e^{-\int_{0}^{t} r_{s} d s} d \hat{I}_{s}\right]<\infty$ for $\hat{I}$ as given by 19 .

With this assumption, one can repeat almost verbatim the proof for the finite horizon case.

Theorem A.3 Under Assumption A.2, there always exists one unique optimal investment plan $I^{*}$ for the infinite-horizon sequential irreversible investment problem.

\section{B Proof of Theorem 3.4}

Proof: It is sufficient to check the first order conditions (5) and (6). Let $I^{*}$ denote the investment plan that finances $C^{l, \delta}$. From $(7)$, the gradient at time $t$ is given by

$$
\nabla_{t} \Pi\left(I^{*}\right)=e^{\delta t} \mathbb{E}\left[\int_{t}^{\hat{T}} e^{-(r+\delta) s} \pi_{c}\left(X_{s}, C_{s}^{l, \delta}\right) d s \mid \mathcal{F}_{t}\right]
$$

As $C^{l, \delta}$ tracks the level $l$, the marginal profit of investment is rewritten as

$$
\nabla_{t} \Pi\left(I^{*}\right)=e^{\delta t} \mathbb{E}\left[\int_{t}^{\hat{T}} e^{-(r+\delta) s} \pi_{c}\left(X_{s}, e^{-\delta s} \sup _{u \leq s}\left(l_{s} e^{\delta s}\right)\right) d s \mid \mathcal{F}_{t}\right]
$$

Trivially, it holds true that

$$
\sup _{u \leq s} l_{s} e^{\delta s} \geq \sup _{t \leq u \leq s} l_{s} e^{\delta s}
$$

Moreover, as marginal profit is decreasing, it follows with the help of the backward equation that

$$
\nabla_{t} \Pi\left(I^{*}\right) \leq e^{\delta t} \mathbb{E}\left[\int_{t}^{\hat{T}} e^{-(r+\delta) s} \pi_{c}\left(X_{s}, e^{-\delta s} \sup _{t \leq u \leq s}\left(l_{s} e^{\delta s}\right)\right) d s \mid \mathcal{F}_{t}\right]=e^{-r t}
$$

This proves (5). When $d I_{t}^{*}>0$, the process $\left(l_{s} e^{\delta s}\right)_{s \in[0, \hat{T}]}$ reaches a new running maximum at time $t$, that is,

$$
l_{t} e^{\delta t} \geq l_{u} e^{\delta u} \quad \text { for all } u<t
$$


In this case, we have

$$
\sup _{u \leq s} l_{s} e^{\delta s}=\sup _{t \leq u \leq s} l_{s} e^{\delta s}
$$

which leads to

$$
\nabla_{t} \Pi\left(I^{*}\right)=e^{\delta t} \mathbb{E}\left[\int_{t}^{\hat{T}} e^{-(r+\delta) s} \pi_{c}\left(X_{s}, e^{-\delta s} \sup _{t \leq u \leq s}\left(l_{s} e^{\delta s}\right)\right) d s \mid \mathcal{F}_{t}\right]=e^{-r t}
$$

Clearly, (6) is also satisfied by $I^{*}$. 